\title{
LA LECTURA Y LA ESCRITURA DE LOS TEXTOS ACADÉMICOS EN EL TALLER DE CONFRONTACIÓN DE LA LICENCIATURA EN EDUCACIÓN FÍSICA DE LA UNIVERSIDAD PEDAGÓGICA
} NACIONAL: “Un ir tras las huellas" documentales

THE READING AND WRITING OF ACADEMIC TEXTS IN THE CONFRONTATION WORKSHOP OF THE DEGREE IN PHYSICAL EDUCATION OF THE NATIONAL PEDAGOGICAL UNIVERSITY: a "go after the tracks" documentaries

\section{Lilia Cañón Florez ${ }^{2}$}

\section{Debbie Brajel Rzonzew Fernández ${ }^{3}$}

Universidad Pedagógica Nacional

$1 \quad$ Este artículo forma parte de los hallazgos y resultados de las investigaciones "Entre concepciones y prácticas docentes, a propósito del texto académico en el Taller de Confrontación de la Licenciatura en Educación Física" avaladas por la Subdirección de Gestión de Proyectos de Investigación de la Universidad Pedagógica Nacional -CIUP- (2017) y "El texto académico en el Taller de Confrontación: Concepciones de los estudiantes del Programa de Licenciatura en Educación Física." avaladas por la Subdirección de Gestión de Proyectos de Investigación de la Universidad Pedagógica Nacional -CIUP- (2018). 


\section{Lilia Cañón Flórez²}

\section{Debbie Brajel Rzonzew Fernández ${ }^{3}$}

\section{RESUMEN}

Este artículo coloca en escena un "ir tras las huellas" teóricas e investigativas en relación con la lectura y la escritura de textos académicos en la formación universitaria de docentes, en un espacio académico particular: el Taller de Confrontación de la Licenciatura en Educación Física de la Universidad Pedagógica Nacional

\section{ABSTACT}

This article puts on the stage a theoretical and investigative "going after the tracks" in relation to the reading and writing of academic texts in the university education of teachers, in a particular context: the Confrontation Workshop of the Degree in Physical Education of the National Pedagogical University.

PALABRAS CLAVE: lectura, escritura, texto académico, formación de docentes y Educación FÍSICA.

KEYWORD: reading, writing, academic text, teacher training and Physical Education.

\section{PUNTOS DE PARTIDA: A MANERA DE INTRODUCCIÓN}

Este artículo de revisión, producto de investigación, expone algunos trayectos teóricos e investigativos relacionados con la lectura y la

$2 \quad$ Magister en Lingüística Española. Profesorainvestigadora. Facultad de Educación Física. Universidad Pedagógica Nacional Facultad de Ciencias de la Educación. Universidad de La Salle.

https://orcid.org/0000-0003-2316-1002

https://scholar.google.es/citations?user=Q9oacLcAAAAJ\&h $\underline{\underline{l=e s}}$

3 Candidata a Magíster en Desarrollo Educativo y Social. Fundación Centro Internacional de Educación y Desarrollo Humano -CINDE-. Universidad Pedagógica Nacional. Participación en monitoría de investigación Subdirección de Gestión de Proyectos Universidad Pedagógica Nacional -CIUP-Durante el pregrado. (2016). Coinvestigadora egresada. Evaluadora Colciencias Programa Ondas. https:// orcid.org/0000-0001-7054-7261 escritura de textos académicos en la formación universitaria de docentes; a su vez, manifiesta un balance de los procesos investigativos propios en torno a este objeto de estudio (avalados por la Universidad Pedagógica Nacional); y, sitúa el texto académico en la perspectiva curricular de la Licenciatura en Educación Física (LEF) y al Taller de Confrontación (TC). Valga señalar que todo intento por compilar recorridos documentales no puede atribuirse pretensiones de totalidad. En la trazabilidad emergente de los procesos de investigación, este artículo entrecruza y concatena resultados de las investigaciones: "Entre concepciones y prácticas docentes, a propósito del texto académico en el Taller de Confrontación de la Licenciatura en Educación Física" y "El texto académico en el Taller de Confrontación: Concepciones de los estudiantes del Programa de Licenciatura en Educación Física." avaladas por la Subdirección de Gestión de Proyectos de Investigación de la Universidad Pedagógica Nacional -CIUP- (2017-2018).

Estos proyectos situaron su pregunta problema en las concepciones de texto académico manifiestas por estudiantes y docentes participantes en el Taller de Confrontación (TC) de la Licenciatura en Educación Física de la Universidad Pedagógica Nacional. Se planteó, entonces, como propósito principal describir las concepciones de texto académico manifiestas por los estudiantes en formación y por los docentes en sus prácticas en el contexto del TC.

De acuerdo con este objetivo, se privilegió el enfoque de investigación cualitativo de corte descriptivo dada su validez cuando se pretende la construcción de conocimiento en las ciencias sociales, en especial cuando se aplica al fenómeno educativo.

Se puede señalar, entonces, que dos centros fundamentarían las actividades de investigación: recoger la información necesaria y suficiente y estructurarla en un todo pertinente, cohesionado, coherente y adecuado. En consecuencia, uno 
de los momentos investigativos se dedicó a la profundización teórica, puesto que esta etapa permite determinar cómo se ha tratado el tema acerca del cual se investiga, reconocer cómo se encuentra en el ámbito académico e identificar las tendencias teóricas que se han aproximado a la temática. Se trata, entonces, de un "ir tras las huellas" del objeto de estudio que posibilita sistematizar información para proyectarla como sustrato investigativo.

\section{DE LA LECTURA, LA ESCRITURA Y EL TRASEGAR POR LOS TEXTOS ACADÉMICOS EN LA FORMACIÓN UNIVERSITARIA DOCENTE}

La lectura y la escritura de textos académicos en la universidad, halla un lugar preponderante en las producciones investigativas de la profesora Gloria Rincón Bonilla (2003), en "Aprender con y sobre los textos en el aula" se declara que la lectura se convierte en una experiencia significativa porque va más allá de los procesos informativos. Por eso, esta experiencia solicita el acompañamiento de los docentes; es decir, no puede dejarse que el estudiante, simplemente, lea, pues se perdería la razón de ser de la lectura; tampoco, se trata de solo crear hábitos porque se dejaría de lado la capacidad analítica y, aún más importante, la reflexiva acerca de lo leído. En definitiva, la experiencia en la lectura no está puesta en la cantidad sino en la reflexión y la vivencia de los textos académicos que, además, se deben diferenciar de los textos cotidianos o para el ocio.

Desde esta investigación se ratifica que la lectura debe convertirse en una experiencia con una intencionalidad previa. En tal sentido, no puede desplegarse como un mandato para cumplir, exclusivamente, con los parámetros académicos. Al respecto, los estudiantes universitarios necesitan guías de sus maestros para dar vida a la lectura de textos de acuerdo con los avances en su formación. En definitiva, para Rincón Bonilla (2003), la lectura se transforma en experiencia cuando pasa a planos de significación y genera análisis competentes y de corte no solo lingüístico sino semiótico de los textos académicos. Desde ahí, se abren las ventanas de la escritura.

En relación con esta perspectiva semiótica, el profesor Fabio Jurado (1998), de la Universidad Nacional de Colombia, hace un señalamiento respecto de su uso en la formación docente. Refiere, que esta formación continua a la par del accionar docente; por eso, no termina al finalizar los programas académicos. Las acciones del aula, como procesos semióticos, hacen parte de las lecturas que el docente efectúa de su realidad. En palabras de María Eugenia Dubois (2011), la formación docente se encuentra ligada al amor por la lectura. Se trata de la lectura para saber ser y saber hacer y, por supuesto, para el ser docente, el saber saber. Sin importar el contexto o las opciones curriculares, en el docente se ara el amor por la lectura, sin importar el área específica de trabajo. Está visión de potenciar la lectura, demuestra, para la autora, la verdadera responsabilidad social de los formadores; en especial, cuando se dedican a "formar formadores".

En los encuentros entre los textos, la formación docente y los procesos educativos e investigativos, la profesora Narváez (2009), señala que la lectura de textos retroalimenta su construcción; por lo tanto, los procesos investigativos se encuentran ligados a la comprensión y la producción de textos. De esta manera, interpela acerca de las lecturas y de las producciones textuales que se les sugiere y se les solicita a los profesores en formación. Desde el contexto académico se entrelaza la formación como investigador; de ahí, la necesidad de configurar capacidades en investigación a partir de los textos, sus comprensiones, sus usos y sus construcciones. Sin embargo, a los docentes en formación, enfatiza la profesora, no se les enseña a hacer una metalectura de 
los contenidos porque la preocupación recae en la interpretación de conocimientos disciplinares y se dejan de lado otras concepciones y las interconexiones entre distintas disciplinas.

En estas conexiones entre texto académico y formación universitaria, la profesora Nylza García Vera (2008) observa que la escritura constituye la representación coherente del discurso. De ahí que, de la elaboración de los procesos correspondientes sobre lectura, entra en juego la capacidad de producción; especialmente, en la universidad, crear textos académicos depende del buen uso de la lectura y de los procesos de comprensión. Se colocaría, entonces, en un orden cognitivo la expresión del discurso desarrollado por la lectura:

No se trata entonces de enseñar la lectura como una técnica aislada que resuelva el problema de la comprensión de los textos académicos. Se trata más bien de entender que si los conceptos previos, la visión de mundo, los intereses, las emociones, los afectos, los saberes y el conocimiento sobre el propio lenguaje escrito, están implicados en la construcción del sentido de estos textos, de lo que se trata aquí es de pensar las condiciones de posibilidad que en términos pedagógicos tenemos para ampliar este conjunto experiencial de los estudiantes (p. 86)

En este orden de ideas, se concentran las copiosas investigaciones acerca de la alfabetización académica y de la inaplazable tarea de hacerse cargo de los estudiantes inexpertos- en sus procesos de lectores y escritores en los ámbitos universitarios; en específico, por parte de los docentes como expertos en el desentrañamiento de los textos propios de la academia superior (Carlino, 2003). También, desde Daniel Cassany (2008), hace presencia la cultura letrada como concepto englobante de las prácticas letradas no solo desde la academia sino, también, en todos los espacios cruzados por lo sociocultural.

Al respecto, el término literacidad ha tomado relevancia al otorgar pluralidad al manejo de los géneros escritos por parte de las diversas comunidades. La literacidad cubre la utilización de los respectivos códigos, los géneros textuales, las funciones del discurso, los roles de los participantes en los actos de lectura y escritura y, en general, los valores, sentimientos, pensamientos, intenciones y formas de actuación discursiva y de desenvolvimiento textual en comunidad, en convivencia:

En la vida muchos eventos letrados son actividades repetidas y regulares que, a menudo, pueden ser útiles como ápices para la investigación en el campo de la literacidad. Algunos eventos se encadenan en secuencias de rutina que pueden ser parte formal de los procedimientos y expectativas de instituciones sociales como los lugares de trabajo, las escuelas y las agencias de bienestar social. Ciertos eventos son estructurados gracias a expectativas y presiones más informales, como las que se dan en el hogar y en los grupos de compañeros y colegas. Los textos son una parte crucial de los eventos letrados, de tal forma que el estudio de la literacidad es en parte un estudio de textos, de la manera como se han producido y se han usado. (Barton, D. y Hamilton, M., 2004, p. 114)

En torno a precisiones respecto del texto académico, Uribe (2007), en "La enseñanza de la composición escrita de textos expositivoexplicativos. Presupuestos teóricos y propuesta didáctica dirigida a profesores de programas no humanísticos de la Universidad del Quindío", reflexiona acerca de las metodologías de escritura que siguen los docentes en formación y de la construcción de textos académicos relevantes para su saber disciplinar. Los 
hallazgos investigativos evidenciaron, entre otros asuntos, que para los estudiantes las asignaturas especializadas en la lengua materna se consideraban poco pertinentes $y$, más bien, ocasionan pérdidas de tiempo que debería dedicarse a materias más significativas.

En este sentido, el profesor Alfonso Cárdenas Páez (s.f) -líder del Grupo ELECDIS- afirma:

Nunca como ahora, se había visto que el lenguaje fuese un factor decisivo en el desarrollo educativo. (...) Sin embargo, existe desequilibrio con respecto a su aplicación pedagógica al español como lengua materna: de un lado, la enseñanza se ha orientado hacia la formación teórica sistemática: del otro, y en contraste con el matiz descriptivo y explicativo del punto de vista anterior, se ha propagado una docencia normativa, directiva y memorística (...). (s.p.)

Lo anterior encuentra enlace cuando se indica que: "(...) se evidencia la necesidad asumir los procesos de la lectura y la escritura más allá del componente gramatical (...) concepción que implica (...) asumir el reconocimiento de las relaciones existentes entre la lengua y los contextos socioculturales (...)" (Medina Bejarano y Cañón Flórez, 2007, p. 123). Se muestra, entonces, en acuerdo con Uribe, la necesidad de concatenar acciones que propicien intersecciones entre las asignaturas dedicadas al uso de la lengua materna con las disciplinares, a través de la escritura de textos de corte expositivo-explicativo en tanto que su estructura corresponde a las exigencias comunicativas de carácter científico.

Frente a concepciones y representaciones de los docentes en el marco de sus actividades con la lectura y la escritura, las profesoras Douglas, López y Padilla en su publicación titulada "Argumentación y pensamiento crítico articulados en la alfabetización académica: reflexiones docentes" (s.f.), dan reconocimiento a la necesidad de generar planes de capacitación docente basados en el reconocimiento de las representaciones y las reflexiones metacognitivas de las actividades con la lectura y la escritura que promueven los docentes en las aulas universitarias.

En un campus particular, el artículo de González Pinzón, Salazar-Sierra, Molina Ríos, y MoyaChaves (2015) "Acciones para la construcción de una política de lectura y escritura universitaria", producto de la investigación "Formación inicial en lectura y escritura: de la educación media al desempeño académico en la educación superior" que adelantaron trece equipos de investigación de distintas universidades colombianas -conformados por docentes pertenecientes al comité de investigaciones de RedLees- presenta los resultados correspondientes a la Pontificia Universidad Javeriana-Bogotá (PUJ). En ella se resalta la importancia de la enseñanza de lo que se denomina el campo educativo y el fortalecimiento de la "Lengua materna" como herramienta para la interpretación, la argumentación y la construcción de textos académicos. Del mismo modo, se aduce que los procesos obligatorios de enseñanza y fortalecimiento de la lengua materna podrían -o no- mejorar las capacidades de los estudiantes para la interpretación de textos académicos.

Se trae a colación la intencionalidad de crear una cultura para los estudiantes universitarios de, forma tal, que puedan relacionarse $y$ comprender los textos académicos; es decir, involucrarse en una cultura académica más formalizada a través del estudio de este tipo de textos. No obstante, a pesar de los esfuerzos de las universidades por implementar mecanismos de enseñanza que conlleven la inmersión en esta cultura, pareciera que las metodologías empleadas no han sido suficientes para los estudiantes universitarios porque, en primer lugar, no todos provienen de un mismo contexto 
educativo ni poseen las mismas capacidades de involucramiento con la lengua materna; $y$, en segunda instancia, las intencionalidades de involucrarse con textos académicos pueden variar de estudiante a estudiante: algunos se quedan en una formación técnica de los saberes más que una acción reflexiva acerca de lo leído.

En relación con la construcción de textos académicos, en este estudio se muestra que los estudiantes hacen producciones para desempeñarse con la tarea o el trabajo por presentar, pero no logran compenetrarse con la lectura y la escritura como herramientas más allá de elementos para la tarea académica. Otro punto de tensión, en este artículo, se refiere al paso del estudiante de la Educación Media (EM) a la universidad. Se argumenta que en la formación profesional aparecen otros componentes que se deben enfrentar al momento de leer los textos solicitados en la educación superior, pues se necesita comprender con significado y sentido para construir nuevos pensamientos y conocimientos. Se sustenta que, en la EM, la lectura gira alrededor de textos literarios -que tampoco se desglosan con suficiencia- y se olvida el trabajo con otros tipos de textos. Esta situación genera pocas concordancias entre los procesos de lectura y escritura de la EM y las exigencias de la cultura letrada universitaria. Se destaca que, ante la complejidad de enfrentar la lectura y la escritura en las disciplinas respectivas a cada programa de formación, “(...) independientemente de la carrera de estudio los estudiantes asisten a los cursos de lectura y escritura inicial como una oportunidad para fomentar y potenciar las competencias [que permitan] enfrentar todas las exigencias de las aulas universitarias" (González Pinzón, et al, 2015).

Desde este espacio, para potenciar la comprensión y la producción del texto académico, se otorga preponderancia a una "(...) práctica docente reflexiva que permita cambiar visiones tradicionales sobre el aprendizaje, el rol del estudiante y sobre la lectura y la escritura en la universidad. Vale la pena retomar la reflexión sobre el docente universitario, profesional que en muchos casos carece de formación pedagógica" (González Pinzón, et al, 2015, s.p.).

En este contexto de ubicación de la lectura, la escritura, el texto académico y las prácticas docentes universitarias, el profesor Fernando VásquezRodríguez(2013)-quien cuenta conuna amplía producción investigativa relacionada con estos textos y sus didácticas en la universidadtrae la atención hacia la complejidad de "contar con las estrategias y el tacto necesario para que otros puedan [aprender]". Esto permite "apreciar la compleja urdimbre de relaciones y situaciones en las que está inmerso el acto de enseñar".

En un espectro de amplitud hacia la universidad del país, la investigación de Pérez Abril y Rincón Bonilla (Coord.) (2013) “¿Para qué se lee y se escribe en la universidad colombiana? Un Aporte a la consolidación de la cultura académica del país", expone de manera detallada, entre otros aspectos, tendencias de las prácticas de la lectura y la escritura en la universidad colombiana y concepciones académicas relacionadas con las interacciones con los textos académicos. Al respecto, se enfatiza que estos textos sirven para fortalecer el quehacer propio de cada área para la cual está destinado este texto; por eso, su uso en la universidad debe trascender el contenido técnico y atender el desarrollo de los problemas epistémicos que cada saber solicita. También, se sustenta que las formas como se desenvuelve la comprensión de los textos académicos se relacionan con el quehacer y la manera de asumir la didáctica por parte de los docentes.

Por su parte, Rubiela Aguirre (2008), frente a las actuaciones de los docentes, llama la atención acerca del uso de técnicas de motivación para promover la lectura y la escritura de los estudiantes. Se espera alcanzar 
un acercamiento más interesado a los procesos lectores y escritores; sin embargo, la autora insiste en la necesidad de tener en cuenta las variaciones que pueden surgir por los distintos contextos donde provienen los estudiantes. A su vez, recalca la presencia de los textos que circulan a través de las tecnologías de la información y la comunicación, pues generan otras formas de leer y describir que conllevan adaptaciones y cambios en las prácticas que los docentes realizan a través de la lectura y la escritura.

Finalmente, vale la pena indicar que respecto de concepciones y prácticas de la lectura y la escritura en la universidad, el grupo de investigación en Didáctica de la Lengua Materna y la Literatura (DiLeMa), como parte de los productos derivados de la investigación "Análisis de una muestra representativa de los relatos presentados al Concurso Nacional de Cuento Gabriel García Márquez" exponen un balance, como estado del arte, referido a 35 investigaciones en: "Estado del arte de las concepciones sobre prácticas de lectura y escritura en la universidad colombiana" (Camargo Martínez, et al., 2008). Para cada una de las 35 investigaciones citadas, se sintetizan problemas, marcos teóricos, metodologías y resultados con el propósito de plasmar tendencias, tensiones, perspectivas y desafíos que, desde los trabajos descritos, se plantean a las prácticas de los y las profesoras en la educación superior.

\section{LA LICENCIATURA EN EDUCACIÓN FÍSICA Y EL TALLER DE CONFRONTACIÓN EN LA UNIVERSIDAD PEDAGÓGICA NACIONAL}

El Taller de Confrontación (TC) corresponde al séptimo espacio de la estructura curricular de la Licenciatura en Educación Física. Su estructura de trabajo colegiado entre docentes y estudiantes lo convierte en un espacio académico representativo de la Licenciatura. Por eso, ocupa un lugar privilegiado en su currículo:
(...) en este espacio se da la posibilidad de encuentroy reconocimiento de diálogo (entre maestros y estudiantes) de los espacios de formación a partir de la reflexión sobre la relación entre la acción corporal directa (los talleres) y el desarrollo temático de las áreas (argumentación teórica, interiorización de la acción corporal directa). El taller de Confrontación como espacio académico en el P-LEF, está presente en todo el proceso formativo desde primer a décimo semestre. Son dimensiones del taller de confrontación: formación académica profesional, transdisciplinariedad, investigación formativa y práctica educativa, desarrollo y sistematización del trabajo final denominado Proyecto Curricular Particular (PCP). (...) El trabajo que aquí se realiza se hace posible con la participación conjunta de estudiantes y maestros que indagan sobre las semejanzas y diferencias de los objetos de estudio, sentidos y significados de las prácticas corporales en diferentes culturas y momentos históricos, sociales, económicos y políticos. (Documento de Autoevaluación, 2014, pp. 9-10).

Desde la concepción del TC, el aula de clase se convierte en un lugar donde convergen distintas tensiones que atraviesan una conjugación de saberes, unas posturas pedagógicas y unas maneras de asumir lo didáctico que involucran, entre otros aspectos, lo ideológico y lo sociocultural. Aquí, emergen, también, las complejidades de la interacción entre los participantes delTC. Enesteespacio, laformación de docentes se configura en un intercambio de saberes, de didácticas y de concepciones, pues, su realización se lleva a cabo con la presencia de docentes representativos de las distintas áreas que componen el currículo de la Licenciatura y con la participación de los estudiantes, quienes presentan los avances en su formación a través de prácticas de lectura y escritura que se concretizan en textos académicos. 
De ahí que, los procesos de enseñanza y aprendizaje inherentes a este contexto hacen surgir no solo inquietudes cognitivas -y metacognitivas- sino también preguntas asociadas a la formación y el manejo de textos, al considerar a la lectura y la escritura no solo como tareas académicas sino como "prácticas culturales" (tal como lo sustenta Rockwell, 2001, p. 7 al mencionar los trabajos de Chartier 1995; De Certeau 1996) el objeto de enseñanza académica de estas se convierten un eje fundamental en la formación docente ya que, "(...) las formas culturales que norman las situaciones y la interacción pueden influir en las prácticas de lectura y escritura (...)" (Rockwell, 2001, p. 8). Frente a esto Carlino (2006), plantea la urgencia y la necesidad de enseñarles a los futuros docentes los modos de leer y escribir en su saber disciplinar porque estos resultan transformándose en los instrumentos esenciales para los aprendizajes específicos de su campo conceptual y de formación.

Si el TC cumple en la práctica lo que en el espíritu de su concepción subyace, se abrirían puertas para generar vínculos entre equipos de docentes y estudiantes. En términos de Echeverri y Rodríguez (2013), lograr concretar el TC desde una perspectiva de equipo propiciaría.

- Las relaciones entre diversas ciencias y saberes unidos por afinidades en la construcción de sus objetos, de conocimientos, de métodos y de sus campos de experiencia y experimentación.

- La necesidad de investigar problemáticas comunes planteadas desde los interrogantes que surgen en la construcción del plan de estudios y de los proyectos de investigación del núcleo (...). (p. 13).

En definitiva, el TC conforma un espacio propicio para analizar las problemáticas en los distintos ámbitos y niveles de formación, con base en los aportes de las teorías y las prácticas pedagógicas de los espacios académicos de la LEF, que encuentra un lugar de expresión privilegiado en textos académicos. Como producto de los TC, se sistematiza la experiencia tanto de los espacios teóricos como de los talleres a través de la producción de un texto académico -implica su lectura y comprensión-.

En los resultados de la investigación "Análisis crítico de las prácticas pedagógicas relativas a la formación del gusto en la educación del cuerpo en la Licenciatura de Educación Física", el profesor Andrés Castaño (2008), indica la imperante necesidad de reconocer el gusto por la lectura y la escritura en la formación de pregrado en Educación Física. Al respecto, se manifiesta que, en estos contextos educativos, las tendencias hacia una rápida profesionalización conllevan pasar de afán por la lectura y la escritura. Lo anterior se acentúa cuando se olvida que el trabajo pedagógico con la lectura y la escritura debe trascender hacia la autonomía que, entre otros, conforma uno de los ejes fundamentales de formación en la LEF:

Lo anterior se válida aún más si se tiene en cuenta que el Proyecto Curricular Licenciatura en Educación Física se direcciona hacia la formación de un profesional de la Educación Física crítico, reflexivo, con pensamiento complejo, investigador, democrático, ético, y autónomo. Las características señaladas anteriormente se entienden en el proceso de formación del profesional como la construcción de un sujeto que [entre otros]: Crea conocimientos, productos y procesos referidos a la educación, la pedagogía y la didáctica y sus relaciones con la Educación Física. (Proyecto Curricular Licenciatura en Educación Física, 2014, p. 7).

En múltiples ocasiones, el afán de acumular información impide detenerse en trabajos con los textos que potencien la comprensión y la creación. En esta creación, el dilucidar 
e interrelacionarse con los textos implica interrogarse alrededor de las actividades propuestas y utilizadas para su comprensión y su producción. De ahí que:

En el ejercicio que orienta el proceso educativo en relación con la lectura para su comprensión y asimilación, el docente se encuentra con dificultades tales como: el fomento de la lectura técnica que sólo compete al saber específico y no se fomenta a partir de allí, el ejercicio de preguntas, sino que se privilegia el ejercicio de la respuesta. A la comprensión se impone la memorización y se olvida de la reflexión sobre lo leído. (Castaño y otros, 2008).

Lo anterior enmarca el lugar del texto académico en el TC de la Licenciatura en Educación Física y enlaza las palabras de Rosa Julia Guzmán Rodríguez (2012) cuando afirma que escribir un texto académico requiere que el estudiante, "aprenda a escribir lo que sabe y produzca conocimiento que pueda ser compartido con las comunidades académicas, como corresponde a los procesos de formación de los estudiantes en el nivel universitario" (p. 6). Por esto, para Cañón Flórez y Caballero Páez (2016) llevar a cabo el proceso de escritura del Proyecto Curricular Particular (trabajo de grado -PCP-) como texto académico:

(...) le posibilita al estudiante descubrir nuevos aspectos $y$, de este modo, retomar, darle sentido y complejizar el acto educativo. Asimismo, adelantar una investigación que establezca con claridad sus paradigmas, métodos, estrategias, técnicas de investigación y marcos teóricos de referencia en los que encuentra una mediación básica o fundamental en un proyecto de investigación que busca situar en contextos educativos particulares y en problemáticas específicas, diversos saberes pedagógicos, didácticos, investigativos, disciplinares y epistemológicos (s.p.).
Lo anterior cimienta el lugar preponderante del textoacadémico en los procesos de formaciónque se desenvuelven en el TC y que se entrecruzan con el currículo general de la Licenciatura. De ahí que las concepciones, el texto académico, sus comprensiones y sus usos sitúan una organización de ideas que han construido históricamente tanto los docentes como los estudiantes, a través de su formación y de sus prácticas. Desentrañar estas concepciones implica el análisis de las situaciones problema imbricadas en el texto académico, los cambios cognitivos, las creencias y las maneras como lo expresan. En suma, como lo afirma Vásquez Rodríguez (2007), "La formación, [configura un espacio donde se conjugan...], nuestras maneras de hacer o decir, nuestras diferencias" (p. 32).

El texto académico localizado en el TC y en sus proyecciones en la formación de licenciados abre puertas para preguntar por sus formas discursivas, sus registros de lengua, la activación de estrategias y las maneras, como desde ciertos rasgos textuales, se llega a su comprensión y producción; y también, por el estudiante y el profesor como lector y escritor de estos textos y sus modos de hacerlos.

\section{Aquí vale traer a colación que:}

Ubicarse en la formación universitaria de docentes implica asumir la lectura y la escritura de textos académicos como una manera de apropiar el conocimiento y, además, de exponer tal consecución. Se manifiesta, entonces, la importancia de la comprensión y la producción textual. Por eso, de manera constante se plantean actividades que se proyectan hacia la comprensión y se solicitan labores que, desde la escritura, posibilitan dar cuenta de los avances en el proceso formativo y hacer evidente la suficiencia académica y, por supuesto, investigativa. (Cañón Flórez y Cifuentes, 2015). 
Se puede aducir, además, que, si la formación de docentes en Educación Física se encuentra atravesada por la pedagogía, las investigaciones y reflexiones acerca de la formación y la educación, conllevan otorgar un lugar preponderante a la lectura, la escritura y los textos académicos como ejes de la formación. Por eso, se requieren docentes que indiquen estrategias o pautas para leer y para escribir $y$ de estudiantes que se interroguen frente a tales estrategias. Al respecto, el profesor Daniel Fernández (2008) revela que los programas de formación en Educación Física solicitan, con urgencia, incluir actividades dirigidas al desarrollo de la lectura y la escritura que, en ningún momento, van en detrimento de las prácticas físicas, sino que más bien dan lugar a su reflexión.

Por su parte, Daniel Racciote (2013), evidencia que, de manera constante, los profesores manifiestan sus inquietudes frente a la lectura y la escritura de los docentes en formación. Refiere, al respecto, que la preocupación no puede recaer en el déficit sino en el estudio de las prácticas de lectura y escritura de los estudiantes y en la reflexión acerca de la responsabilidad de los profesores como formadores.

Se espera que cada profesor, en su quehacer pedagógico, entre a reflexionar acerca de la lectura y la escritura y sus concomitancias con los propósitos de la Licenciatura que aluden, entre otros, a gestar "procesos de construcción disciplinar desde la perspectiva del objeto de estudio asignado al currículo: La experiencia corporal [y a] formar un maestro de educación física con características de científico social del cuerpo" (Proyecto Curricular Licenciatura en Educación Física, 2009). Se puede aducir, entonces, que desde "el ejercicio pedagógico se ha [de buscar] proveer a los estudiantes las herramientas necesarias para dominar el acto de escribir, respondiendo a las características sociales que se transforman con el paso del tiempo" (Cáceres, et al., 2014).

Estas deliberaciones generan preguntas relacionadas con el "equipaje" que arman los futuros docentes para afrontar su quehacer profesional $\mathrm{y}$, allí, asumir sus compromisos frente a la educación en lectura y escritura de sus estudiantes "[Entonces] es necesario que la universidad se replantee el modo en que concreta sus planes de estudio y asuma una visión conjunta, equilibrada, contrastada y coherente de lo que significa ser maestro en general y del papel de éste en relación con la educación en y para la lectura". (Granado, et al., 2007, p.7).

En términos de Carlino (2006) y de Pérez (2008), la formación universitaria acarrea la necesidad de desarrollar capacidades de comprensión crítica, de análisis y de producción de textos que se convierten en las rutas principales para el acceso a la cultura académica y a la comprensión de los discursos de las disciplinas y que, además, originan aprendizajes y toma de posturas frente al conocimiento que, en la formación universitaria de docentes, se convierten en sustratos en las relaciones entre conocimiento-enseñanzaaprendizaje-enseñanza. Esta perspectiva frente a la lectura y la escritura encuentra una concatenación con los propósitos de formación que orientan la Licenciatura en cuanto a Redimensionar la concepción de Educación Física con base en fundamentos científicos filosóficos para transformar la representación social vigente y las praxis del futuro maestro [y] Formar un maestro de educación física capaz de interpretar conceptual, legal y académicamente sus prácticas educativas para tomar decisiones de desempeño profesional contextualizado" (Proyecto Curricular Licenciatura en Educación Física, 2009).

Se hace pertinente enlazar palabras de la investigadora Stella Serrano cuando expresa que en la universidad las prácticas de lectura 
y escritura se entrelazan con las actividades que los estudiantes necesitan cumplir para comprender y construir el conocimiento de las disciplinas. "De ahí que, en la universidad, el estudio e investigación sobre cualquier temática académica, necesariamente debe apoyarse en la comprensión crítica, en el análisis y el procesamiento de las diversas fuentes de conocimiento y en la composición de textos (...)" (Serrano, 2011). Al respecto, la investigadora formula múltiples preguntas relacionadas con la lectura y la escritura en la formación universitaria, vale traer a colación: “¿Cuáles son las experiencias que estamos ofreciendo en cada disciplina para que el estudiante participe en prácticas de lectura y escritura en las cuales se exige este nivel de complejidad?" (Serrano, 2011). Con base en estos cuestionamientos se resalta que, de acuerdo con Marín (2006),

En los estudios universitarios es preciso que se entienda que leer críticamente es una exigencia para que los estudiantes estén en condiciones de procesar información de fuentes diversas y a menudo contrastantes, familiarizarse con las secuencias explicativas y argumentativas en los textos y reconocer posiciones epistémicas (voces en el discurso) no siempre explícitas (...). Estas exigencias precisan un cambio de orientación para abordar la lectura y la escritura en el contexto universitario (...). Es necesario entonces, configurar una pedagogía de la lectura y la escritura críticas para la tarea de formación universitaria (...). Esta reflexión debe conducir a los profesores a transformar la enseñanza e incluir los modos de lectura y escritura que se esperan de los estudiantes para aprender, es decir, para interpretar y producir el discurso propio de los contenidos disciplinares, pues ocuparse de ello es enseñar. (p.31).

\section{DE CONCEPCIONES Y PRÁCTICAS EN LA LECTURA Y LA ESCRITURA DE TEXTOS ACADÉMICOS}

En enlace con lo expuesto, se puede mencionar a Paula Carlino (2013), quien, en sus investigaciones relacionadasconlaalfabetización académica, precisa, en un análisis de trabajos realizados en Argentina, ciertos referentes relacionados con la lectura y la escritura en la universidad. La autora presenta un debate entre la enseñanza situada en las disciplinas y las tendencias a presentar estrategias o formas de trabajar con los textos de manera fragmentaria o determinada por espacios curriculares de especialistas en lengua. Ya no se trata solo de preguntarse por la lectura y la escritura en la educación superior, sino de investigar acerca de "por quién, cómo, dónde, cuándo y para qué hacerlo" (p. 355). Se reformula, entonces, la definición de alfabetización académica, propuesta en la década de los años noventa, por una encaminada a una enseñanza concatenada con las prácticas que la circundan.

La autora enfatiza en la lectura, la escritura y el dominio de los textos académicos como ejes esenciales de la enseñanza en la universidad. Se insiste que estos aspectos deben tratarse en relación con las prácticas de la lectura y la escritura propias de las disciplinas. De ahí las claridades requeridas, durante los procesos de formación, en torno a los textos, sus concepciones, comprensiones y elaboraciones en concatenación con los ámbitos disciplinares. En la formación universitaria no se puede dejar la responsabilidad del trabajo con los textos a espacios curriculares especializados, sino que, por el contrario, deben expandirse hacia "profesores no expertos [... en] tratar aspectos lingüísticos, discursivos y metacognitivos (...)" (Carlino, 2013, p. 360).

Lo expuesto por Carlino indica que reconocer las concepciones de texto académico situadas en "el recorrido como lector en una disciplina 
ayuda a enfrentarse a sucesivos textos sabiendo qué buscar" (Carlino, 2013, p. 360), pues generar estrategias o conceptos de lectura y escritura que no se han enlazado a la disciplina de estudio llevaría a que el "lector podrá sentirse perdido y dispondrá sólo de estrategias generales (leer más lento, saltear, consultar otras fuentes, discutir con otros, releer, etcétera)" (Carlino, 2013, p. 360). Desde una visión de construcción conjunta, se propone generar estrategias y talleres que se armen, de manera colaborativa, con los especialistas de las distintas disciplinas de tal forma que los estudiantes logren desempeñarse como integrantes de su comunidad letrada en cuanto lectores y escritores de disciplinas particulares con conciencia del funcionamiento del lenguaje en estos marcos.

En relación con la formación de docentes en la Licenciatura en Educación Física, el Taller de Confrontación (TC), como espacio académico colegiado, situaría un lugar para "posibilitar que los alumnos se incluyan en situaciones discursivas típicas de comunidades especializadas, según propósitos, significados y valores compartidos. Aprender a leer y a escribir significa formarse para participar y pertenecer a ellas" (Carlino, 2013, p. 361). Se abren puertas para un "trabajo conjunto entre docentes de distintas disciplinas en torno al leer y escribir en las áreas curriculares (...)" (Carlino, 2013, p. 363), lo que brindaría una potenciación para dominar los textos académicos en enlace con los conocimientos disciplinares y pedagógicos propios de la educación física o si, por el contrario, "aparece desaprovechada" [.... Y] Se pierde así la ocasión de formación continua que podría resultar del intercambio entre profesores de escritura y de otras materias" (Carlino, 2013, p. 363).

En enlace con investigaciones relacionadas con concepciones y lo planteado respecto de escribir y leer en las disciplinas y de alfabetización académica Molano y López (2006), adelantaron una investigación, sustentada en estudio de caso, que indagó por las concepciones de profesores y estudiantes acerca de la escritura académica en la Universidad Icesi. Los autores partieron de considerar que las concepciones componen un saber que recae en el quehacer de los profesores, sus formas de enseñanza y los aprendizajes de los estudiantes. Entre otros hallazgos, en esta investigación, se encontró que: las prácticas de escritura giran alrededor de las comprensiones y las producciones de contenido que se concentran en el texto académico; en el trabajo con los textos, las guías de los profesores y las rutas seguidas por los estudiantes todavía distan de tender hacia la formación de comunidad científica, en cuanto "colectividad en la que se utilizan normas de comunicación especializadas para transmitir y evaluar ideas" (p. 127), en especial, porque se acentúa en la apropiación de lo conceptual y no en prácticas discursivas que se corresponden con las disciplinas:

(...) la mayor parte de los estudiantes observados sólo registra en sus respuestas que la escritura sirve para desarrollar contenidos, está mostrando que en la universidad aún se desconocen las otras funciones epistémicas de la escritura y el papel que aporta en la revisión, la transformación y la construcción de los saberes propios de cada disciplina. A pesar de que los estudios actuales convergen en mostrar que lectura y escritura constituyen procesos centrales en cada disciplina, que median en el aprendizaje de nuevas temáticas y en el desarrollo de los conocimientos propios de las distintas áreas de estudio, esto no se observa en las asignaturas técnicas observadas. (Molano y López, 2006, p. 143)

Del mismo modo, se concluye que los estudiantes aducen interés y preocupación 
por aspectos relacionados con la cohesión, la coherencia y la ortografía que no se reflejan en sus producciones escritas. Los investigadores consideran que esto obedece a la insuficiencia de prácticas de escritura en los campos disciplinares que posibiliten la construcción y la expresión del pensamiento científico. La escritura debe convertirse en un reto para el intelecto del estudiante y en una vía para adentrarse en los textos propios de las comunidades disciplinares. Por esto, las concepciones de escritura de los textos académicos en los estudiantes reflejan sus comprensiones y consideraciones respecto de su uso y manera de hacerlos.

También, se señala que, aunque en el Proyecto Educativo de esta Universidad, se expresa la importancia y la relevancia de la producción de textos argumentativos, en la práctica su realización ocupa un mínimo lugar como actividad de escritura. Del mismo modo, se llama la atención respecto de la necesidad de elaborar instructivos, de manera escrita, y de plantear objetivos y estrategias claras para no dejar en el vacío lo que se espera en el trabajo con los textos y sus formas de concebirlos. En definitiva: “¿Qué es lo que realmente esperan los profesores de los trabajos que asignan a sus estudiantes, cuando la gran mayoría no ofrece instructivos suficientemente claros para su elaboración? (Molano y López, 2006, p. 144).

\section{DE LA LECTURA Y LA ESCRITURA DE TEXTOS ACADÉMICOS EN LAFORMACIÓN UNIVERSITARIA DE DOCENTES: UN RECORRIDO DE ANTECEDENTES}

En estos caminos, por la presentación de un balance documental se presentan pretensiones y hallazgos relacionados con proyectos que configuran un itinerario investigativo, respecto de la lectura y la escritura de textos académicos en la formación de licenciados.

En concatenación con lo anterior, se reseñan aquí proyectos que, durante los últimos diez años, han marcado un proceso derivado de la preocupación por el papel de la lectura y de la escritura en la formación de docentes, los textos académicos, la investigación formativa y la elaboración de trabajos de grado (PCP). Durante la vigencia 2009, se desarrolló el proyecto concerniente con la comprensión lectora de los textos académicos en el ámbito universitario. (Cañón Flórez y Acevedo, 2009). En su momento, se señaló que antes de proponer vías de solución a partir de supuestos problemas en procesos de comprensión lectora para llegar a resultados específicos, se hace necesario reconocer, desde la concepción de la lectura como proceso interactivo y transaccional, no solo el déficit del lector frente al texto sino más bien las formas como ese lector y los procesos académicos que lo acompañan generan estrategias de comprensión.

En las conclusiones de esta investigación se reconocen los procesos lectores y su relación con las prácticas lectoras de los estudiantes universitarios $\mathrm{y}$, a su vez, da lugar a pensar formas de construir y reconstruir actos de enseñanza que, por supuesto, sugerirían conexiones con perspectivas pedagógicas y propuestas curriculares concretas. Asimismo, se expresaron interrogantes derivados de la riqueza de las voces de los estudiantes y de los profesores que se confrontan y se enfrentan en actos lectores y en comprensiones de lectura que se pueden tomar como una mirada frente a interrogantes particulares de las investigaciones adelantadas y que dan apertura a diseñar otros proyectos relacionados con la lectura en formación universitaria de docentes; por ejemplo: ¿hasta dónde se propician procesos interactivos, intencionales, constructivos y de inferencias en los procesos académicos de formación de docentes?

Al mismo tiempo, al interrogar por las estrategias de acuerdo con las fuentes de lectura propuestas por los docentes se encontró una mínima 
referencia a artículos provenientes de revistas académicas y especializadas. De ahí, entonces, se derivó la inquietud frente al artículo científico -como texto académico- su uso y las estrategias propuestas para su comprensión. Desde este cuestionamiento, se realizó el proyecto "El artículo científico como texto académico en el proceso de formación investigativa de los estudiantes de los pregrados de la Facultad de Educación de la Universidad Pedagógica Nacional" (CIUP, 2011). Al ubicar lo "académico", se encontró que sus productos comunicativos se estructuran desde las exigencias propias de su denominación: textos académicos. Las disposiciones discursivas y de estructuración de estos textos implica el dominio de patrones de significado reconocidos por las comunidades científicas y académicas. Estas disposiciones, muestran al artículo científico como un texto académico. (Cañón Flórez y Rodríguez, 2010).

Entonces, la lectura de un artículo científico solicita la activación de estrategias de comprensión que lleven hacia el logro de conocimientos y al alcance de la supervisión y de la regulación del propio proceso cognitivo $\mathrm{y}$, también, propicien el acercamiento a la investigación. De ahí que la pregunta problema planteó si "los ejercicios investigativos derivados de la elaboración de trabajos de grado en la formación en investigación de futuros docentes, los profesores de la Facultad de Educación de la Universidad Pedagógica Nacional generan la lectura comprensiva de artículos científicos" (Cañón Flórez y Rodríguez, 2010). En términos generales, se pudo evidenciar que muy pocos trabajos reportan el uso del artículo científico como un referente teórico de consulta. Se encontró, además, que las licenciaturas establecen lineamientos para la elaboración de los trabajos de grado; pero, en múltiples ocasiones, tales directrices terminan en la prescripción.

La lectura de artículos científicos significaría una oportunidad para activar estrategias y mecanismos de comprensión que permitan el logro de conocimientos; se posibilitaría, también, la supervisión y la regulación del propio proceso cognitivo y el acercamiento a procesos investigativos; $y$, en un proceso que podría denominarse "de reacción en cadena", otorgaría fundamentos cognitivos e instrumentales para la construcción de trabajos de grado.

La revisión de los trabajos de grado implicó la observación detenida de los RAE y la observación tangencial de ciertos elementos relacionados con los procesos de síntesis y de síntesisanálisis adelantados para su elaboración. Esto llevó a preguntarse por los caminos académicos y de ejercicios de investigación recorridos en el proceso de formación docente para la lectura y la escritura de los denominados textos de síntesis y de síntesis-análisis.

Se ubicó en el escenario la pregunta por las estrategias cognitivas y metacognitivas que propician los docentes y a las cuales recurren los estudiantes para adentrase en las exigencias de comprensión lectora y de construcción de textos de síntesis y de síntesis-análisis en la elaboración de marcos teóricos o de estados del arte en el marco de desarrollo y presentación de trabajos de grado.

Se adelantó, entonces, el proyecto "Entre-textos universitarios: estrategias de lectura y escritura en el marco de la construcción de un proyecto de grado" -CIUP, 2012-. En esta propuesta (Cañón Flórez y García, 2011) se planteó que la elaboración de un trabajo de grado exige asumir paradigmas y métodos de investigación, pero, más allá, su construcción demanda un paso ineludible relacionado con la elaboración de marcos teóricos o de estados del arte, o bien de los dos. Se programó indagar por los esquemas y las disposiciones que guían la manera de abordar la lectura y la producción de resúmenes, reseñas y raes (como textos sintéticos y sintético-analíticos) en el proceso de 
construcción de marcos teóricos o estados del arte. Asimismo, se interrogó por el papel del tutor en este proceso -desde su lugar de experto- y de cómo propicia el reconocimiento y el uso de estrategias que conlleven mayores niveles de apropiación, comprensión y producción de documentos a partir del tipo de textos aludido.

Durante los años de 2013 y 2014, se adelantó el proyecto relacionado con el ensayo académico. La pregunta de investigación giró alrededor de la lectura y la escritura de textos de corte argumentativo como el ensayo académico.

Entre los hallazgos encontrados, se puede aducir que el ensayo académico, al ubicarse en una tipología argumentativa, implicaría el seguimiento de ciertos requerimientos cuando se solicita y se elabora. Por eso, su escritura se demandaría cuando se considere que se ha pasado por la elaboración de otro tipo de textos y se cuente con suficiencia de conocimiento temático, lingüístico y discursivo para producir textos que sostengan una postura personal y puedan recurrir a formas de argumentación que lleven hacía la persuasión o el convencimiento.

En estos quehaceres investigativos germinó el proyecto "De textos y estrategias: a propósito de la lectura y la escritura en los Proyectos Curriculares Particulares -PCP- de la Licenciatura en Educación Física" (Cañón Flórez y Caballero Páez, 2016) con la pretensión de tipificar la producción académica y el ejercicio investigativo expuesto en la construcción de $\mathrm{PCP}$, en consonancia con su articulación con EI Proyecto Curricular Licenciatura en Educación Física. En síntesis, se puede acotar que este "ir tras las huellas" investigativas abre puertas para proponer interrogantes que, desde los insumos producidos, atraen la reflexión y el estudio de la lectura y la escritura hacia otros ángulos de la formación universitaria.

Estainvestigación (UPN, CIUP, 2016) relacionada con el PCP considera que, con el acopio de fuentes documentales y su comprensión, junto con las orientaciones del profesor experto, supone que se elaborarán textos que concretan la escritura y las comunicaciones, producto de los ejercicios de investigación y de la confrontación en diferentes contextos de la práctica pedagógica y educativa. Por eso, formuló la pregunta problema respecto de las estrategias de lectura y escritura propuestas por los docentes y utilizadas por los estudiantes, para construir este trabajo final de grado.

En el año 2017, se desarrolló una investigación situada en las concepciones de texto académico desplegadas en las prácticas docentes de los profesores y las profesoras de la Licenciatura en Educación Física de la UPN (Cañón Flórez; Medina Bejarano y Caballero Páez, 2017). En el 2018, la investigación planteó un detenimiento en las concepciones de texto académico manifiestas por los estudiantes de la citada licenciatura (Cañón Flórez; Medina Bejarano y Caballero Páez, 2018).

\section{ASPECTOS DE CIERRE: A MANERA DE CONCLUSIONES}

\section{De la lectura, la escritura y el texto}

La explicación del proceso de producción de textos ha cobrado vital singularidad a partir de que la lingüística ha abordado el texto como una integralidad, como una unidad comunicativa. Desde esta perspectiva, se han procurado las más diversas explicaciones o modelos que han sido sintetizados y analizados por Álvarez y Ramírez (2006). Estos autores hacen énfasis en la amplitud y en la complejidad de modelos o teorías que, al atender el asunto de la escritura, asumen posturas socio-cognitivas, textuales e incluso didácticas que se han pretendido a la hora de encontrar elementos para el fortalecimiento de la construcción de textos.

Si bien tales modelos no han tenido en principio una finalidad didáctica, sí se pueden extraer 
estrategias didácticas y posibilidades para el desarrollo de la producción escrita en las aulas; en especial cuando se encuentra que los modelos analizados presentan convergencias muy importantes como la relevancia del contexto, el desarrollo de estrategias cognitivas y metacognitivas para la producción escrita, la mirada de la escritura como un proceso complejo que requiere de entrenamiento, la secuencia de etapasofasesfundamentalesparalaconstrucción -planificación, textualización, revisión y edición, por ejemplo- y el peso específico de la revisión. De igual manera señalan que la diferencia y la complejidad textuales están en concordancia con la audiencia destinataria, el propósito de producción, el estatus del autor y el contenido de la información, entre otros aspectos.

Los anteriores aspectos enmarcan la complejidad que rodea al texto académico, en especial, cuando su lectura y escritura se despliegan, precisamente, para adentrarse en una alfabetización académica. En este caso, se hace necesario tener en cuenta que la actividad de lectura en este nivel superior es distinta, pues "los modos de indagar, de aprender y de pensar en un área de estudio [están] vinculados con las formas de leer y de escribir que hemos ido desarrollando dentro de la comunidad académica a la que pertenecemos" (Carlino, 2005, p. 13). Se considera, así, que la mediación pedagógica o la enseñanza de estrategias de lectura en la educación superior no puede asumirse como menos substancial que en la educación básica o en la secundaria.

La formación de un lector en la universidad implica su inserción en una cultura discursiva propia de las disciplinas y de las comunidades científicas a las cuales aspira pertenecer mediante el estudio de una carrera. En concordancia con Carlino, se trataría, en rigor, de una alfabetización académica. Así, se espera que, desde la inserción en una cultura académica, alcance el lugar de lector-escritor. Esta experticia, para el caso de la formación de docente, toma una doble vía por cuanto no solo se trata de la experticia en una disciplina sino también en la experticia de saber enseñarla y ésta se encuentra cruzada por el dominio de los textos académicos que le competen. En tanto experto o representante de tal comunidad académica, el profesor dispone y necesita conocer acerca de los repertorios bibliográficos propios de su disciplina o campo profesional; por ello, puede "aportar hojas de ruta y lentes que ayuden a recorrer y enfocar lo que, según nuestro conocimiento de la disciplina, es importante en los textos" (Carlino, 2005, pp. 71-72).

Desde el aspecto comunicativo, los textos académicos conllevan acercarse, en un proceso de formación de educación superior, a rasgos textuales que, desde la comprensión de cierto tipo de texto -por ejemplo, un artículo académico-, llevaría a la lectura de otros textos relacionados y a la producción textual. Cobra relevancia, aquí, retomar la literacidad, cuando Cassany y Castellá (2010) se plantea que:

El concepto de literacidad abarca todos los conocimientos y actitudes necesarios para el uso eficaz en una comunidad de los géneros escritos. En concreto, abarca el manejo del código y de los géneros escritos, el conocimiento de la función del discurso y de los roles que asumen el lector y el autor, los valores sociales asociados con las prácticas discursivas correspondientes, las formas de pensamiento que se han desarrollado con ellas, etc. (p. 1).

Por otra parte, se torna imposible hacer referencia al texto, sin traer a colación la escritura anclada en la difusión del conocimiento y las investigaciones que atañen a las disciplinas; en especial, puestas en prácticas relacionadas con aquello que ocurre en los contextos de la educación. Al respecto, la obra de Tolchinsky y Simó. (2001), puesta en la lectura y la escritura a través del currículo, sustenta la 
necesidad de enseñar la escritura a los diversos grupos mediante las distintas asignaturas que corresponden a un currículo. Esto ubica la exigencia en el reconocimiento de los textos que soportan las asignaturas y también los modos de llevarlos al aula; es decir, solicitan del docente su identificación y manejo.

Valga resaltar que la propuesta de "escribir a través del currículo", muestra una tradición anglosajona, que toma fuerza en la década de los años ochenta, derivada de WAC (writing- across- curriculum); Sus orientaciones argumentan que la escritura constituye el pilar fundamental del aprendizaje y su uso no puede limitarse a las asignaturas dedicadas a la enseñanza de la lengua. De ninguna manera, considera este enfoque que la escritura pueda abordarse fuera de los temas implícitos a cada asignatura, pues la escritura se toma como el método para pensar por excelencia.

Esta postura, al colocarse en la universidad, solicita involucrarse en los conceptos de las disciplinas en concatenación con los patrones comunicativos y de construcción textual que las representan.

En un detenimiento frente a "escribir a través del currículo", Carlino (2004), sustenta que la escritura no puede quedar en manos de especialistas, sino que debe ser asumida por los docentes en su cátedra, puesto que aquí la escritura

[...] alberga un potencial epistémico, es decir, no sirve sólo para registrar información o comunicarla a otros, sino que puede ser un instrumento para acrecentar, revisar y transformar el propio saber. Por otra parte, en nuestras facultades es frecuente la queja de los profesores acerca de cómo redactan sus alumnos, pero también es habitual que no se consideren responsables de hacer nada a partir de esta comprobación.
Las palabras de Carlino, otorgan complejidad al trabajo con la lectura, la escritura y los textos en la universidad. Esto solicita que el docente reconozca cada texto como un constructo potenciador de lo epistémico, lo experiencial y lo comunicativo. El docente no solo sabe de su disciplina, sino también de cómo se enseña a través de los textos.

Los textos, las maneras como se organizan y sus marcas discursivas conforman esquemas estructurales, formales o textuales; en otros términos, se requieren esquemas cognitivos previos esenciales para la construcción del significado del texto. Aunque tales esquemas formales, en parte, se adquieren por la experiencia con una diversidad de textos que circulan en el entorno, se espera que los docentes, en atención al trabajo interdisciplinario -base del actual modelo de educación superioraseguren, mediante un trabajo sistemático, estrategias de lectura y de escritura de las distintas modalidades que componen los tipos textos -entre ellos, los académicos-.

Los cambios históricos, respecto de los tipos de texto, recaen en las maneras como se usan, en tanto mediaciones, en lo didáctico y en las formas como se potencializan desde la enseñanza. Las concepciones de los textos afectan las formas como se comprenden y se producen y, también, como se conciben y se didactizan en las prácticas docentes.

En tal sentido, y en relación con aspectos pragmáticos, el texto académico se manifiesta en producciones orales, escritas o audiovisuales en el marco de las actividades científicas y universitarias que responden a la intención de producir o transmitir conocimientos sistemáticos de alcance social y cuyos destinatarios son los miembros de las distintas comunidades científicas y universitarias en los ámbitos local, nacional o internacional. Al traer a colación a Bajtín, se ubica al texto académico en correspondencia con un género discursivo 
secundario que presenta diferencias frente al primario por cuanto el uso de los géneros discursivos primarios discurre en la cotidianidad y se mediatizan en la oralidad (2002, pp. 248293).

Se desprende, así, como Cassany y otros autores, consideran al texto académico como un tipo de discurso altamente elaborado, caracterizado por el uso del registro formal de la lengua y por utilizar un léxico preciso y específico (citado por Tapia y otros, 2003). Desde la sociología, de acuerdo con Bernstein (1993), el texto académico se sitúa en correspondencia con los códigos elaborados que se agencian en prácticas discursivas propias de un contexto; en el caso de la universidad, se elaboran desde corpus epistemológicos y desde la circulación de los conocimientos característicos de estas agencias de socialización. Esto conlleva el rompimiento con los códigos restringidos que transitan en la oralidad cotidiana perteneciente a contextos particularistas y locales.

En este sentido, el texto académico requiere una selección de formas más esmeradas, lo cual implica mayor esfuerzo por parte del sujeto hablante (Tapia y otros, 2003). En cuanto a los esquemas de formulación, Cubo de Severino (2005) destaca en los textos científicos coincidencias como la desagentivización, las nominalizaciones y el léxico específico. Estas características llevan a hablar de estrategias o procedimientos generales para la construcción y deconstrucción de textos académico-científicos.

Cubo de Severino (2005) indica que los textos académicos presentan patrones de significación reconocidos por la comunidad científica y que pueden clasificarse de acuerdo con criterios de agrupación: textos de difusión en ámbitos académicos y científicos, textos de control y aval de investigadores y textos de estudio de disciplinas. Desde lo formal, un texto académico puede estructurarse mediante cualquiera de los códigos y medios disponibles para la comunicación: oral -una conferencia, por ejemplo-, escrito -un artículo o libro- y audiovisual -un video, entre otros-

Así, comprender y escribir textos académicos resignifica, otorga sentido y conlleva la consolidación de una cultura académica propia de este ámbito de formación. Lo anterior enmarca posibilidades de convertir estos textos en temas de estudio que pueden derivar en distintos problemas: su comprensión y producción; sus disposiciones discursivas y textuales; su representatividad de culturas académicas; sus implicaciones en un lector-escritor; y sus repercusiones en ámbitos particulares de elaboración, comprensión y circulación, entre otros. Estas redes de problemas entretejen la complejidad de estudiar el texto académico.

\section{REFERENCIAS BIBLIOGRÁFICAS}

Aguirre, R. (2008). Fomentar la lectura y la escritura en estudiantes de formación docente. Acción Pedagógica, 17, 86 95.

Bajtín, M. (2002). Estética de la creación verbal. Buenos Aires: Siglo XXI.

Barton, D. y Hamilton, M. (2004). La literacidad entendida como práctica social. En V. Zavala., M.

Bernstein, B. (1993). La estructura del discurso pedagógico. Madrid: Morata.

Cáceres, H. et al. (2014). Devenir histórico de enseñanza de la lectura y la escritura en Colombia. Bogotá: Universidad de San Buenaventura.

Cárdenas Páez, A. (1998). Hacia una pedagogía integral del lenguaje. Documento digitalizado. Bogotá: Universidad Pedagógica Nacional. 
Camargo Martínez, Z. y otros. (2008). Estado del arte de las concepciones sobre prácticas de lectura y escritura en la universidad colombiana. Grupo de Investigación en Didáctica de la Lengua Materna y la Literatura (DiLeMa). Armenia, Colombia: Universidad del Quindío.

Cañón Flórez, L. y Acevedo, S. (2009). Estrategias cognitivas y metacognitivas de comprensión lectora de los textos académicos en el ámbito universitario. Proyecto de investigación. UPNCIUP. Convocatoria interna para grupos con proyectos de investigación en educación y pedagogía vigencia 2009 .

Cañón Flórez, L. y Rodríguez, C. (2010). El artículo científico como texto académico en el proceso de formación investigativa de los estudiantes de los pregrados de la Facultad de Educación de la Universidad Pedagógica Nacional. Proyecto de investigación. UPN-CIUP. Convocatoria interna para grupos con proyectos de investigación en educación y pedagogía vigencia 2011.

Cañón Flórez, L. y García, N. (2011). Entretextos universitarios: estrategias de lectura y escritura en el marco de la construcción de un proyecto de grado. Proyecto de investigación. UPN-CIUP. Convocatoria interna para grupos con proyectos de investigación en educación y pedagogía. Vigencia 2012.

Cañón Flórez, L. (2013-2014). De los textos académicos en la formación universitaria de docentes. Bogotá: Universidad Pedagógica Nacional

Cañón Flórez, L. y Cifuentes, R. (2015). De recorridos teóricos y de posibilidades didácticas en torno al ensayo académico en la formación de docentes. Simposio
Internacional de Educación, Pedagogía y Currículo. Ponencia presentada en el Simposio Internacional de Educación, Pedagogía y Currículo. Red Iberoamericana de Pedagogía Redipe-, Chile.

Cañón Flórez, L. y Caballero, F. (2016). De textos y estrategias: a propósito de la lectura y la escritura en los Proyectos Curriculares Particulares -PCP- de la Licenciatura en Educación Física. Proyecto de investigación. UPN-CIUP. Convocatoria interna para grupos con proyectos de investigación en educación y pedagogía. Vigencia 2016.

Cañón Flórez, L; Caballero, F. y Medina, R. (2017). Entre concepciones y prácticas docentes, a propósito del texto académico en el Taller de Confrontación de la Licenciatura en Educación Física. Proyecto de investigación. UPN-CIUP. Convocatoria interna para grupos con proyectos de investigación en educación y pedagogía. Vigencia 2017.

Cañón Flórez, L.; Medina, R. y Caballero Páez, F. (2018). El texto académico en el Taller de Confrontación: Concepciones de los estudiantes del Programa de Licenciatura en Educación Física." Subdirección de Gestión de Proyectos de Investigación de la Universidad Pedagógica Nacional -CIUP. Proyecto de investigación. UPNCIUP. Convocatoria interna para grupos con proyectos de investigación en educación y pedagogía. Vigencia 2018.

Carlino, P. (2003). Leer textos científicos y académicos en la educación superior: Obstáculos y bienvenidas a una cultura nueva. Uni-Pluri/Versidad, 3 (2), 1-9.

Carlino, P. (2004). El proceso de escritura académica: Cuatro dificultades de la 
enseñanza universitaria. Educere, 8 (026), 321-327.

Carlino, P. (2005). Escribir, Leer y Aprender en la Universidad. Una Introducción a la Alfabetización Académica. Buenos Aires: Fondo de Cultura Económica.

Carlino, P. (2006). Escribir, leer y aprender en la universidad. Buenos Aires, Argentina: Fondo de Cultura Económica

Carlino, P. (2013). Alfabetización académica diez annoys dispose. Revista Mexicana de Investigación Educativa, 18 (57), 355-381. (ISSN: 14056666)

Castaño, A. (2008). Análisis crítico de las prácticas pedagógicas relativas a la formación del gusto en la educación del cuerpo en la licenciatura de Educación Física. Pedagogías formativas del gusto por la lectura y la escritura. Medellín: Centro de investigaciones CICIDEP del Instituto de Educación Física de la Universidad de Antioquia.

Cassany, D. (2008). Prácticas letradas contemporáneas. México: Ríos de Tinta

Cassany, D. y Castellá, J. (2010). Aproximación a la literacidad crítica. PERSPECTIVA, Florianópolis, 28, (2), 353-374.

Cubo de Severino, L (Coord.). (2005). Los textos de la ciencia. Córdoba, Argentina: Comunicarte.

De Certeau, M. (1996). La invención de lo cotidiano. Tomo I. Artes de Hacer. México: Universidad Iberoamericana.

Douglas, S., López, E. y Padilla, C. (s.f.). Argumentación y pensamiento crítico articulados en la alfabetización académica: reflexiones docentes. En L Laco., L. Natale., y M. Ávila. (Comp.), La lectura y la escritura en la formación académica, docente y profesional (pp. 45-60). Argentina: Editorial de la Universidad Tecnológica Nacional edUTecNe.

Dubois, M. E. (2011). La lectura en la formación y actualización docente: comentario sobre dos experiencias. Legenda, 15, 64-79.

Echeverri, J. y Rodríguez, H. (2013). Equipo docente: formación, cultura colaborativa e interdisciplinariedad. Pedagogía y Saberes, 39, 11-20.

Fernández, D. (2008). Lectura, Escritura y Educación Física. Revista Digital educación Física y Deportes, 13 (119). Recuperada de http://www.efdeportes. com/

Facultad de Educación Física. (2009). Documento Proceso de Autoevaluación con fines de Acreditación de Calidad. Facultad de Educación Física. Universidad Pedagógica Nacional.

Facultad de Educación Física. (2014). Documento Proceso de Autoevaluación con fines de Acreditación de Calidad Facultad de Educación Física. Universidad Pedagógica Nacional.

García Vera, N. (2008). Lectura, escritura, cultura académica y formación de docentes. Nodos y nudos, 3 (25), 81-89.

González Pinzón, B. Y., Salazar-Sierra, A., Molina Ríos, J. A. y Moya-Chaves, S. (2015). Acciones para la construcción de una política de lectura y escritura universitaria. Folios (41), 143-155.

Granado, C. (2007). Los futuros maestros y maestras ante la educación lectora hábitos lectores, creencias, capacitación adquirida y necesidades 
formativas de los futuros maestros y maestras respecto a la educación lectora. Proyecto de investigación Pacto Andaluz por el Libro, Empresa Pública de Gestión de Programas Culturales. Consejería de Cultura de la Junta de Andalucía, España.

Guzmán Rodríguez, R.J. (2012). Escritura académica en la universidad. Bogotá. Universidad de a Sabana. Halliday, M.A.K. (2001). "On the grammatical foundations of discourse". R. Shaozeng, W. Guthrie and I.W. Ronald Fong. (Eds.) Grammar and Discourse: proceedings of the International Conference on Discourse Analysis (pp.47-58). Macau: University of Macau Publications Centre.

Jurado, F. (1998). Investigación, escritura y educación. Bogotá: Plaza y Janes / Editores Colombia.

Marín, M. (2006). Alfabetización académica temprana. Lectura y Vida, 4 (27), 30-38.

Medina Bejarano, R. y Cañón Flórez, L. (2007). Las concepciones de la lectura y la escritura en la labor del docente de educación superior. Pedagogía $y$ Saberes, 27, 117-126.

Molano, L. y López, G. (2006). Concepciones de profesores y estudiantes sobre la escritura académica en la Universidad Icesi. Revista Lenguaje, 35 (1), 119-146.

Narváez, E. (2009). Comprender y Producir Textos Escritos para Investigar. Cali: Universidad Autónoma de Occidente.

Pérez Abril, A. M. (2008). Leer y escribir para tomar posición frente al saber. Análisis de prácticas de lectura y escritura académicas en la universidad. Encuentro Nacional e Internacional sobre Lectura y Escritura en Educación
Superior. Ponencia presentada en el Encuentro Nacional e Internacional sobre Lectura y Escritura en Educación Superior. REDLEES y la Asociación Colombiana de Universidades, Bogotá, Colombia.

Pérez Abril, A. M. y Rincón Bonilla, G. (Coords.). (2013) ¿Para qué se lee y se escribe en la universidad colombiana? Un aporte a la consolidación de la cultura académica del país. Bogotá. Pontificia Universidad Javeriana.

Racciote, D. (2013). Estudiantes y escritores. Un nuevo suelo textual para la formación docente en Educación Física. EFDeportes.com, Revista Digital, 18 (185). Recuperada de http://www. efdeportes.com/

Rockwell, E. (2001). La lectura como práctica cultural: conceptos para el estudio de los libros escolares. Educação e Pesquisa. Faculdade de Educação da Universidade de São Paulo. Disponible en http://dx.doi.org/10.1590/S151797022001000100002 .

Rincón Bonilla, G. (2003). Aprender con y sobre textos en el aula. Cali: Publicaciones Universidad del Valle.

Serrano, S. (2011). Lectura crítica y escritura argumentativa para tomar posición frente al conocimiento disciplinar en la formación universitaria. Entre Lenguas, 16, 27-41.

Tapia, M., Burdiles, G. y Arancibia, B. (2003). Aplicación de una pauta diseñada para evaluar informes académicos universitarios. Revista Signos, 36. 
Tolchinsky, L. y Simón, R. (2001). Escribir y leer a través del curriculum. Barcelona: Universidad de Barcelona. ISBN 8485840-90-9.

Uribe, G. (2007). La enseñanza de la composición escrita de textos expositivo-explicativos. Presupuestos teóricos y propuesta didáctica dirigida a profesores de programas no humanísticos de la Universidad del Quindío. (Tesis Doctoral). Universidad Complutense de Madrid, España.

Vásquez Rodríguez, F. (2007). Educar con Maestría. Bogotá: CMYK.

Vásquez Rodríguez, F. (2013). El quehacer docente. Bogotá, Colombia: Universidad de La Salle. 